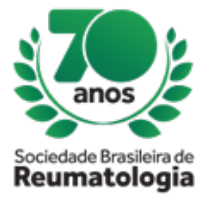

\title{
SECONDARY MYELOFIBROSIS INDUCED BY SARCOIDOSIS: CASE REPORT
}

Allana Campos Alves (UFMT, Sinop, MT, Brasil), Diogo Rios de Avila (UFMT, Sinop, MT, Brasil), Andressa Gabrielly Rodrigues Beserra (UFMT, Sinop, MT, Brasil), Gabriel Freitas de Campos (UFMT, Sinop, MT, Brasil), Fabiana de Castro Machado (UFMT, Sinop, MT, Brasil), Gabriel Ribeiro Leão Barroso (UFMT, Sinop, MT, Brasil), Heloisa Maria Lopes Scarinci (UFMT, Sinop, MT, Brasil), Ivana de Menezes (UFMT, Cuiaba, MT, Brasil), Flavio Fernandes Barboza (UFMT, Sinop, MT, Brasil)

\section{BACKGROUND}

Sarcoidosis is a chronic multisystemic inflammatory disease characterized by non-caseating granulomas formation. Its etiology is unknown, but the most accepted hypothesis involves the genetic predisposition of the patient, in association with human leukocyte antigen (HLA) and exposure to environmental factors (moisture, organic and inorganic particles, and infections).

The disease can affect several organs, the most common being lung, lymph nodes, skin, and eyes. Bone involvement is less common, affecting 3-13\% of patients with sarcoidosis, and bone marrow is even more rarely affected. Patients with bone marrow involvement may present with hematological abnormalities such as anemia, leukopenia and thrombocytopenia as a consequence of secondary myelofibrosis. Differential etiologies for this condition are multiple myeloma (MM), systemic lupus erythematosus, mycobacteria and fungi.

\section{CASE REPORT}

A 52-year-old hypothyroid female patient was referred to the hematologist with intense weakness for the last two years and loss of $16 \mathrm{~kg}$ in the last year. For one year she had been receiving treatment for iron deficiency anemia, diagnosed in a basic health unit, without improvement. Admission laboratory tests showed anemia, elevated inflammatory markers and kidney failure(table1).

Due to diagnostic suspicion of $\mathrm{MM}$, bone marrow biopsy was performed, revealing extensive fibrosis aplasia of approximately $90 \%$ of the erythroid lineage -, chronic inflammatory process, granulomas and asteroid bodies(figure1). Immunohistochemistry proved negative for neoplasms and culture was negative for mycobacteria and fungi. The patient was then referred to the rheumatologist for suspected sarcoidosis. Computed tomography (CT) of the chest identified discrete fibrosing interstitial lung disease.

Considering the biopsy findings of granuloma and the exclusion of infectious or neoplastic etiologies, pulmonary involvement and probable renal failure associated with hypercalcemia, the diagnosis of sarcoidosis was determined.

Due to renal failure and hematological clinical condition, $40 \mathrm{mg}$ prednisone therapy was started, yielding satisfactory laboratory response in 30 days and recovery of the previous weight in 60 days(table2).

Because of hematologic prognosis caused by extensive fibrosis, we decided to initiate therapy with azathioprine $2 \mathrm{mg} / \mathrm{kg}$ associated with progressive withdrawal of corticosteroids.

\section{CONCLUSION}

Myelofibrosis due to sarcoidosis is poorly described in the literature, its treatment lacks further evidence and is often performed empirically in order to achieve satisfactory clinical outcome. Atypical diagnosis is important to emphasize that the rheumatologist must consider differential diagnoses of secondary myelofibrosis, in order to investigate all possible etiologies. 